\title{
Internal Gravity Waves Spectrum Generated by a Cylindrical Body Moving in a Stratified Fluid
}

\author{
M. Akbarnejadbaie ${ }^{1}$, A. A. Bidokhti ${ }^{2 \dagger}$, M. Akbarinasab ${ }^{3}$, M. Ezam $^{4}$ and S. Allahyaribeik ${ }^{4}$ \\ ${ }^{1}$ Department of Physical Oceanography, Science and Research Branch, Islamic Azad University, \\ Tehran, Iran \\ ${ }^{2}$ Institute of Geophysics University of Tehran, Tehran, Iran \\ ${ }^{3}$ Department of Environmental and Marine Sciences, University of Mazandaran, Babolsar, Iran \\ ${ }^{4}$ Department of physical oceanography, Science and Research Branch, Islamic Azad University, \\ Tehran, Iran \\ †Corresponding Author Email: bidokhti@ut.ac.ir
}

(Received September 19, 2019; accepted December 28, 2019)

\begin{abstract}
Internal waves are abundant in stratified environments such as the atmosphere and the ocean. In this paper, the internal waves and turbulent wake effect of a cylindrical body in a linear stratified environment is investigated. In a glass tank with dimensions $3 \times 1 \times 0.5(\mathrm{~m})$, a linear stratification with buoyancy frequency $(\mathrm{N})$ equal to 0.51 per second, was set up. Then a cylindrical body with $6 \mathrm{~cm}$ in diameter and $45 \mathrm{~cm}$ at length was towed in the fluid, using a computer controlled system. While changing the Froude (from 0.16 to 1.5 ) and Reynolds numbers of the flow, the effects of these changes were examined on the formation of the internal waves and wake of the cylinder. The internal waves generated were studied using shadowgraph technique and signal fluctuations were recorded. The results show that the presence of internal waves depend on changes of buoyancy frequency $(\mathrm{N})$, Froude numbers, and Reynolds numbers of the flow. It was also found that with an increase up to the critical Froude number, the activity of internal waves and their wavelengths enhanced. Also, irregular long and short waves as well as turbulence of the environment were observed in the range of supercritical Froude numbers. In this study, the signals are recorded in domains of frequency and statistics. Using an ultra-fast salinity meter (densitometer) for recording signal frequencies it was found that increasing Froude number results in more combination of frequencies occurred in the environment. Based on the results of current experiments and previous studies, an equation was extracted to calculate the wavelength by parameters like velocity of body, maximum frequency value and propagation angle. The energy of wave spectrum increased up to a critical Froude number, and then decreased due to turbulence. The statistical distribution of signals recorded in most of the scenarios was normal.
\end{abstract}

Keywords: Density stratification; Internal waves; Shadowgraph imaging technique; Salinity signal fluctuations; Froude number; Spectral analysis.

\section{NOMENCLATURE}

$\begin{array}{ll}\text { A } & \text { amplitude of oscillation } \\ \text { Anls } & \text { analysis } \\ \text { C } & \text { a constant } \\ \text { D } & \text { length of the body in horizontal direction } \\ \text { F } & \text { external forces } \\ \text { f } & \text { coriolis parameter } \\ \mathrm{f}_{\mathrm{m}} & \text { maximum frequency value } \\ \mathrm{Fr} & \text { Froude number } \\ \mathrm{g} & \text { gravitational acceleration } \\ \mathrm{H} & \text { fluid depth } \\ \mathrm{h} & \text { body thickness } \\ \mathrm{IWs} & \text { intenal waves } \\ \mathrm{K} & \text { wavenumber vector } \\ \mathrm{K}_{\mathrm{h}} & \text { horizontal wave number }\end{array}$

\begin{tabular}{|c|c|}
\hline $\mathrm{N}$ & buoyancy frequency \\
\hline $\mathrm{n}$ & the nth vertical mode \\
\hline $\mathrm{Re}_{\mathrm{e}}$ & Reynolds number \\
\hline $\mathrm{U}$ & velocity of underwater body \\
\hline VDP & Vertical Density Profile \\
\hline $\mathrm{w}$ & vertical velocity \\
\hline WLT & Wave Length In Theory \\
\hline WLE & Wave Length In Experiment \\
\hline$\theta$ & angle of wave number with horizontal \\
\hline$\lambda$ & wavelength \\
\hline$v$ & kinematic viscosity \\
\hline$\rho$ & density \\
\hline$\pi$ & pi \\
\hline$\omega$ & internal wave angular frequency \\
\hline
\end{tabular}




\section{INTRODUCTION}

Nowadays, the study of internal waves (IWs) is one of the most basic studies of oceanography and fluids dynamics, due to its importance with various applications such as estimation of energy transfer and detection. IWs are the factors responsible for the formation of layered structures in marine environments. Schlosser et al. (2019) described vertical transport of energy as one of the functions of IWs and mentioned that wind is a major factor responsible for the formation of these waves; according to them IWs can propagate at least hundreds of kilometers from their source. Regarding the usage of IWs' detection, it should be said that, in the design of underwater bodies, they make them invisible to sound waves and to some extent electromagnetic waves, but the changing hydrophysical parameters of the environment caused by moving underwater bodies cannot be reined. In order to identify them, it can be very helpful to use the changes of the hydrophysical parameters, such as IWs and turbulent wake, created by moving subsurface bodies.

For the first time, Ekman (1904) identified IWs generated by the motion of a ship. Sutherland and Linden (2002), Aguilar et al. (2006), and Bidokhti et al. (2016), have used various methods for formation of IWs by creating glass water channels with different dimensions and they have measured and studied the physical features of generated IWs. Some of these studies have been conducted on the feasibility of specific channels in experimental research.

Numerous studies have been conducted to investigate IWs and turbulent wake in the continuous or stepped stratified fluids. Both in nature and engineering, flow around bodies in the stratified fluid is found in forms of IWs, turbulent wake and dispersion (Thorpe, 2005). Bonneton et al. conducted an experiment to generate IWs in a stratified fluid using a spherical model and found that in a density-stratified environment like the ocean thermocline, the motion of submarine body can generate IWs that can propagate for kilometers (Bonneton et al. 1993). Dotsenko and Savoskin also studied IWs generated by moving a two-dimensional body in a stratified fluid and found out that the interaction of IWs generated by the motion of a body can generate large-scale IWs in the ocean (Dotsenko and Savoskin, 1994). In another experimental study, Stevenson et al. investigated the motion of a cylindrical body in a stratified fluid and observed IWs generated by this motion. They used ray theory to figure out IWs can get trapped in the (stratified part of) fluid (Stevenson et al. 1986). Also, Swain conducted an experimental study and investigated detection techniques of underwater bodies and identified the turbulence wake and IWs generated by the motion of subsurface bodies using optoelectronic methods and he stated that the type of the wake and IWs generated in these environments can depend on the body moving in the environment (Swain and Trinath, 2012). Abdilghanie and Diamessis studied the nonlinear effects of IWs generated by the turbulent wake with numerical modeling (Abdilghanie and Diamessis, 2013). Ohya et al. investigated the wake of a moving cylindrical body in a stratified fluid and reported that the frequency of separation of the vortices from the body and their two-dimensional state changed as density stratification increased (Ohya et al. 2013). Wong and Griffiths also showed the presence of shear currents along with the flow field of IWs in the laboratory due to the motion of a plume, and one of their findings was the dependence of the flow field on the generated stratified structure (Wong et al. 2001). Also, Mitkin and Chashechkin investigated the flow around a circular cylinder moving in a fluid with continuous density stratification and observed the flows associated with IWs generated by the motion of the cylindrical body (Mitkin and Chashechkin, 2000). They showed that the flow mentioned above occurred at low Froude numbers, Fr, which had higher viscosity effects and the perturbation was considerable in front of the body. Glushko et al. studied the turbulent wake of a moving body in a stratified fluid using a mathematical model and they concluded that some fluctuations appear in the wake of a body greater than the average fluid (Glushko et al., 1994). In their experimental study, Meunier et al. examined the effects of IWs generated by the motion of a body in a linearly stratified fluid, and showed that two types of these waves are created: (i) internal waves with long wavelength and (ii) coherent wake waves with short wavelength. They also reported that experimentally the amplitude of the wake waves depended on the Reynolds number (Meunier et al. 2018)

Regarding that the previous studies have not experimented on the received signals from wake and IWs, the objectives of this survey are to: (i) investigate the wake behind a cylindrical body in different Fr numbers in a linearly stratified environment via recording images using the shadowgraph technique and analyzing the recorded signals of the fast-response salinity meter sensor in frequency domain and statistical domain, (ii) study different flow regimes based on the internal waves generated, by taking the physical parameters of the body and the flow into account, and (iii) provide an empirical relation between the speed of the body and internal wavelength by analyzing recorded signals.

\section{SOME THEORY}

The linear theory by Gill is used to show the decay relation of the motion of internal waves in a stratified fluid as follows (Gill 2016; Filonov and Trasviña, 2000; Leder 2002):

$\omega^{2}=N^{2}\left(\frac{K_{h}}{K}\right)^{2}=N^{2} \cos ^{2} \theta$

Where:

$\omega=$ the angular frequency of a wave

$\mathrm{N}=$ buoyancy frequency of the atmosphere

$K_{h}=\left(K_{x}, K_{y}\right)=$ the horizontal wave number 
$K=\left(K_{x}, K_{y}, K_{z}\right)=$ the wave number vector

$\theta=$ the angle between the wavenumber vector and the horizontal direction.

Vertical velocity equation can be obtained from the continuity and the Navier-Stokes equations in the presence of friction and a series of assumptions close to reality (Gill, 2016):

$$
\begin{aligned}
& \frac{\partial^{2}}{\partial t^{2}} \nabla^{2} w+N^{2} \nabla_{h}^{2} w+f^{2} \frac{\partial^{2} w}{\partial z^{2}}-\frac{N^{2}}{g}\left(\frac{\partial^{3} w}{\partial z \partial t^{2}}+f^{2} \frac{\partial w}{\partial z}\right)= \\
& -\frac{1}{\bar{\rho}}\left[f\left(\nabla \frac{\partial F}{\partial z}\right)_{z}+\nabla_{h} \cdot \frac{\partial^{2} F}{\partial z \partial t}-\nabla_{h}^{2} \frac{\partial F z}{\partial t}\right]
\end{aligned}
$$

Where $\mathrm{w}$ represents the vertical velocity and $\mathrm{F}$ denotes the external forces.

If the vertical velocity is assumed to be constant with respect to time and $F \equiv 0,(2)$ can be presented as follows:

$\nabla_{h}^{2} W_{0}-\frac{\omega^{2}-f^{2}}{N^{2}-\omega^{2}}\left(\frac{\partial^{2} W_{0}}{\partial z^{2}}-\frac{N^{2}}{g} \frac{\partial W_{0}}{\partial z}\right)=0$

Where the coefficient $\frac{\omega^{2}-f^{2}}{N^{2}-\omega^{2}}$ determines the type of partial differential equation (PDE). If $\frac{\omega^{2}-f^{2}}{N^{2}-\omega^{2}}<0$

, then (3) is called the elliptic PDE and it implies that the wave motion is impossible due to the absence of a free or common surface. If $\frac{\omega^{2}-f^{2}}{N^{2}-\omega^{2}}>0$, then (3) is called the hyperbolic PDE and it implies that the wave motion is possible; if $N^{2}(z)>f^{2}$ is true for all $\mathrm{Z}$ 's, wave motion is possible when:

$$
f<\omega<N
$$

Therefore, at depth $\mathrm{Z}$ the upper limit for internal wave frequency is determined by the buoyancy frequency $(\mathrm{N})$ and its lower limit by the inertia frequency.

When a body with the average velocity (U) moves in a stratified fluid with buoyancy frequency $(\mathrm{N})$, the wavelength of a wave can be obtained from the following relation:

$$
\lambda=2 \pi \frac{U}{N}
$$

Then in terms of Froude number of the flow with $h$ as body thickness along the vertical direction we can write:

$$
F r=\frac{U}{N h}=\frac{\lambda}{2 \pi h} \quad \text { or } \lambda=2 \pi \cdot h . F r
$$

The wake flow depends on the Reynolds number.

$$
R=\frac{U D}{v}
$$

Where $\mathrm{U}$ is the velocity of the underwater body, $\mathrm{D}$ is the length of a determined body by the diameter of the cylinder or sphere, or the length of the body in horizontal direction and $v$ is the Kinematic viscosity of the fluid.

This shows that the wavelength of generated waves (normalized by h) depends directly on Fr, when there are significant internal waves in flow.

The results obtained from an experimental study performed by Hopfinger et al. on a spherical body showed that the domain of Froude number for internal waves ranged from 0.5 to 4 , and afterwards was dominated by a turbulent wake with irregular waves and substantially smaller wavelengths (Hopfinger et al. 1991).

For stationary internal waves of the body moving in a stratified fluid with a low thickness $h$, considering the boundary conditions in the floor and surface (rigid surface condition), it is shown that the wave has a mode structure (known as normal mode, Gill, 2016) and its structure can be presented as follows (Ohya et al. 2013):

$$
\frac{N H}{\pi U}=\left(n^{2}-k_{x}^{2} \frac{H^{2}}{\pi}\right)^{1 / 2}
$$

Where:

$\mathrm{H}=$ the depth of fluid

$\frac{2 H}{n}=$ the wavelength for the nth vertical mode in the vertical direction.

They also showed that the horizontal wavenumber of stationary wave generated by the body in its moving direction with $k_{x} \longrightarrow 0$ we have:

$$
\frac{N H}{\pi U}=n
$$

Thus, the wavelength of these waves behind the body can be presented as follows:

$$
\lambda=\frac{2^{1 / 2} H}{\left[\left(\frac{N H}{\pi U}\right)^{2}-n^{2}\right]^{1 / 2}}
$$

It is similar to Eq. (6), however, the wave structure in vertical direction is quantized in terms of depth of fluid $(\mathrm{H})$, not body thickness (h) . For $\mathrm{n}=0$ :

$$
\lambda=2^{1 / 2} H \pi F r
$$

Where $F r=\left(\frac{N H}{U}\right)^{-1}$, hence the wavelength of IWs generated can be expressed with respect to fluid depth (if it is relatively less than body thickness) or the thickness of the body.

\section{EXPERIMENTAL METHOD}

\subsection{Linear Stratification in the Lab}

All experiments were conducted in a glass tank with dimensions equal to $3 \times 1 \times 0.5(\mathrm{~m})$. The glass tank was stratified using two tanks of 750 liters equipped with electrical conductivity of saline $(50 \mathrm{mM})$ and fresh 
water (Martin, 1997). Both tanks were evenly filled with water. First, the valve of saline tank was opened, and salt water was poured into the experimental tank using a hose which has a sponge on its outlet within the canal, then the valve between the two tanks was opened immediately. Salt water should be filled and emptied very slowly, so that its layers will not be dispersed and mixed. The experimental tank was filled with water to a height of $80 \mathrm{~cm}$ during about 4 hours, and the accuracy of the depthometer used in the experiments was one millimeter. As the method of filling the tanks and the formation of fluid with continuous density stratification suggests, when salt water in tank 2 was discharged into the experimental tank, fresh water in tank 1 replaced salt water of tank 2, since, as mentioned above, both tanks were evenly filled with water. So as freshwater entered tank 2 , its salt concentration was diluted and a fluid with continuous linear density stratification was formed in the channel. In these experiments the buoyancy frequency $\mathrm{N}$ was 0.51 per second based on the mean gradient of the vertical density profile (VDP). The VDP was obtained by an electrical conductivity meter (EC meter) with an accuracy of 0.01 millisiemens.

\subsection{Characteristics of the Cylindrical Body and Pull System}

A cylindrical body of $6 \mathrm{~cm}$ diameter and $45 \mathrm{~cm}$ length was used to study the internal waves and the wake behind the body. The cylindrical body was controlled by a computer program, and it moved by a cart connected to the computer program in the range of $0.5 \mathrm{~mm} / \mathrm{s}$ to $4.5 \mathrm{~cm} / \mathrm{s}$, and the accuracy of its movement was 0.1 millimeters per second. In order to visualize the wake and the internal waves generated by the motion of the body, an optical method known as shadowgraph was employed.

The body was moved horizontally along the tunnel center, using a set of cart, rail and electric motor controlled by a computer program and its speed was under control. A linear VDP was used in these experiments. Figure 1 shows the schematic representation of the layout of the equipment employed.

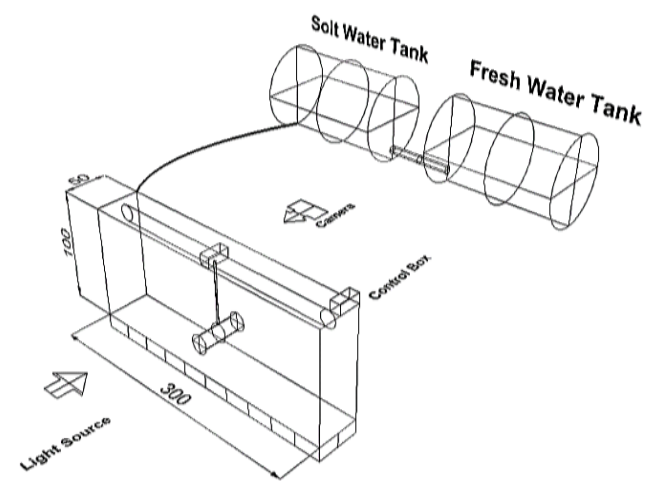

Fig. 1. Schematic showing the flow channel with density stratification and a cylindrical body inside it.

The light source consists of a projector that shines a light from a great distance nearly parallel across the width of the channel. This light forms a shadow of the flow field, illustrating the waves generated by the motion of the cylindrical body, on a thin and opaque paper fastened to the transparent body of the channel. The images of these shadows were recorded by a camera and a camcorder.

After stratifying the channel, buoyancy frequency $\mathrm{N}$ was calculated by measuring temperature and salinity. Figure 2 shows density changes on a vertical direction (quantized by fluid depth) before and after experiments. Buoyancy frequency $(\mathrm{N})$ was $0.51 / \mathrm{s}$ based on the mean gradient of VDP before the experiments. After performing the experiments, $\mathrm{N}$ changed to 0.5 . So, the slight variation in the VDP indicates very little dispersion in the water column.

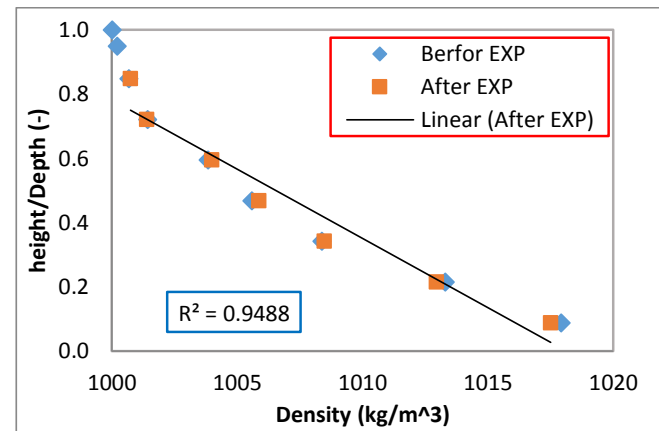

Fig. 2. Vertical density profiles before and after the experiments.

Table 1 shows operating conditions of the experiments along with the length and height of the waves generated by the body at different speeds, after image processing and the frame-to-frame image analyses with respect to different Froude numbers with an error of $\pm 1 \mathrm{~mm}$. No internal waves were detected in velocities of 4 and $4.5 \mathrm{~cm} / \mathrm{s}$ due to a highly turbulent environment.

In this study, the range of Reynolds number was $300 \sim 2700$ and Froude number ranged from 0.16 to 1.5 .

Table 1 Operating conditions along with the length and height of internal waves generated (in cm) using shadowgraph imaging technique

\begin{tabular}{|c|c|c|c|c|c|c|}
\hline $\begin{array}{c}\mathrm{U} \\
(\mathrm{cm} / \mathrm{s})\end{array}$ & $\operatorname{Re}$ & Fr & $\begin{array}{c}\text { WL.T } \\
(\mathrm{cm})\end{array}$ & $\lambda / \mathrm{h}$ & $\begin{array}{c}\text { WL.E } \\
(\mathrm{cm})\end{array}$ & $\begin{array}{c}\mathrm{A} \\
(\mathrm{cm})\end{array}$ \\
\hline 0.5 & 300 & 0.16 & 6.19 & 1 & 6.9 & 0.7 \\
\hline 0.7 & 420 & 0.23 & 8.67 & 1.4 & 10 & 1.1 \\
\hline 1 & 600 & 0.33 & 12.39 & 2.1 & 15 & 2 \\
\hline 1.5 & 900 & 0.49 & 18.58 & 3.1 & 18 & 2.6 \\
\hline 2 & 1200 & 0.66 & 24.77 & 4.1 & 23.5 & 3 \\
\hline 2.5 & 1500 & 0.82 & 30.97 & 5.2 & 35 & 1.4 \\
\hline 3 & 1800 & 0.99 & 37.16 & 6.2 & 39 & 9.4 \\
\hline 3.5 & 2100 & 1.15 & 43.35 & 7.2 & 45 & 3.6 \\
\hline 4 & 2400 & 1.32 & 49.55 & 8.3 & Turb & Turb \\
\hline 4.5 & 2700 & 1.48 & 55.74 & 9.3 & Turb & Turb \\
\hline
\end{tabular}


By the use of shadowgraph technique and image processing and scaling the channel, the length and height of the internal waves behind the body were calculated. It should be noted that all experiments at speeds presented in Table 1 were performed at least twice, which produced approximately the same results. And after closely examining the experiments for each target speed, the length and height of the internal waves were calculated, as shown in Table 1.

A fast-response salinity meter (densitometer) developed by Bidokhti et al. was used to investigate the salinity fluctuations behind the body (Bidokhti and Britter, 2002).

A fast-response salinity meter is a thin tube with outer insulation in which water can enter through the tip of the tube via a tiny hole with plastic block installed to its end and its salinity can be measured up to $500 \mathrm{~Hz}$. The spatial resolution of this device is $1 \mathrm{~mm}$. Through the connections made, the fluctuations of the signals caused by the salinity meter were displayed online on the oscilloscope screen. The distance between sensor and model was $5.2 \mathrm{~h}$ (model diameter, $\mathrm{h}$ ). Since the signals received from the oscilloscope contained noise, the output file was inserted into a software to remove noise. After noise suppression, the extracted signals were analyzed. Using these signals and their diagrams, wave activity and height of the generated IWs were observed and recorded in the table.

Table 2 shows the operating conditions at different Froude numbers and the height of IWs generated by the body at different speeds.

Table 2 Operating conditions including the height of IWs generated (in $\mathrm{cm}$ ) using a fastresponse salinity meter

\begin{tabular}{|c|c|c|c|c|}
\hline $\begin{array}{c}\text { Exp. } \\
\text { No. }\end{array}$ & $\begin{array}{c}\mathrm{U} \\
(\mathrm{cm} / \mathrm{s})\end{array}$ & $\operatorname{Re}$ & Fr & $\begin{array}{c}\text { A (Signal.Anls) } \\
(\mathrm{cm})\end{array}$ \\
\hline 1 & 0.5 & 300 & 0.16 & 0.6 \\
\hline 2 & 0.7 & 420 & 0.23 & 0.89 \\
\hline 3 & 1 & 600 & 0.33 & 1.86 \\
\hline 4 & 1.5 & 900 & 0.49 & 3.3 \\
\hline 5 & 2 & 1200 & 0.66 & 2.73 \\
\hline 6 & 2.5 & 1500 & 0.82 & 4.36 \\
\hline 7 & 3 & 1800 & 0.99 & 4.7 \\
\hline 8 & 3.5 & 2100 & 1.15 & 4.95 \\
\hline 9 & 4 & 2400 & 1.32 & Turb \\
\hline 10 & 4.5 & 2700 & 1.48 & Turb \\
\hline
\end{tabular}

\section{RESULTS AND DISCUSSION}

In this section, the results obtained from experiments on the cylindrical body moving in fluid with continuous density stratification is presented. The buoyancy frequency $(\mathrm{N})$ of the fluid was $0.51 / \mathrm{s}$, and it had very slight changes during the experiments which could be ignored. Figures 3 and 4 show the flow field around the body in a stratified fluid with increasing Froude numbers, recorded by shadowgraph technique. In the figures, it's clear that as Fr increases, the activity of internal waves increases and the wavelengths of these waves become longer. However, when Froude number was greater than 1.32, it resulted in a turbulent environment and thus internal waves could not be seen clearly.

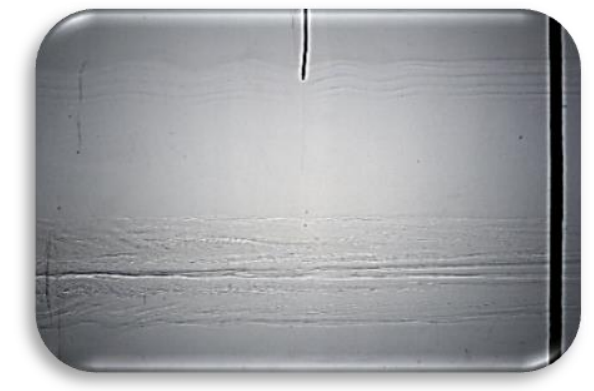

a) $\mathrm{Fr}=0.23$

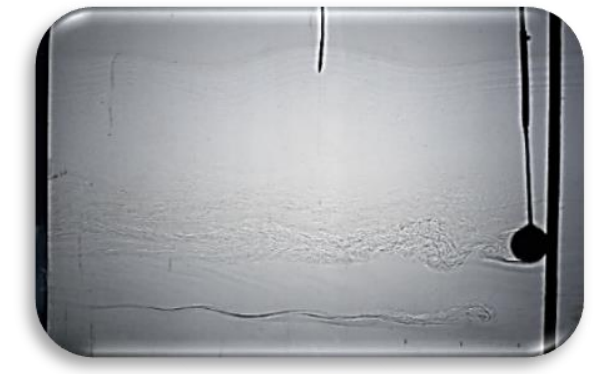

b) $\mathrm{Fr}=0.49$

Fig. 3. Flow field behind the cylindrical body moving in a stratified fluid with $\mathrm{Fr}=0.23$ and $\mathrm{Fr}=0.49$.

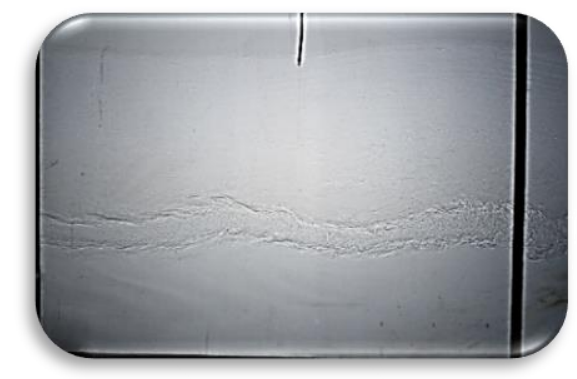

a) $\mathrm{Fr}=0.99$

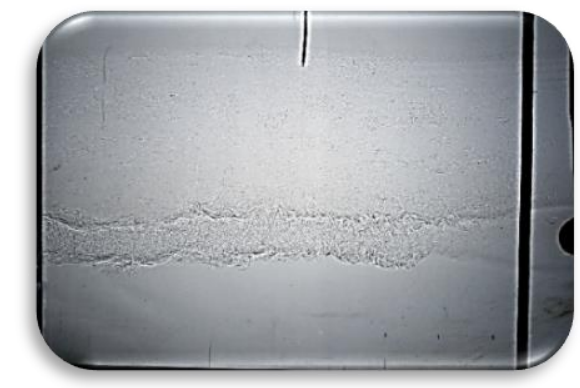

b) $\mathrm{Fr}=1.32$

Fig. 4. Flow field behind the cylindrical body moving in a stratified fluid with $\mathrm{Fr}=0.99$ and $\mathrm{Fr}=1.32$. 


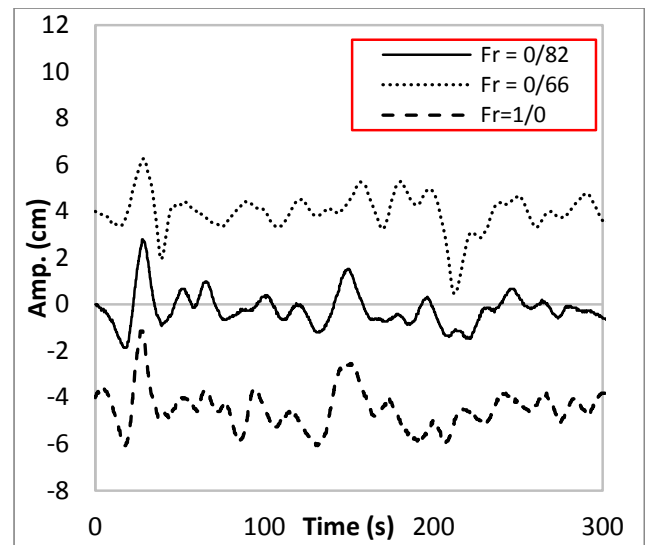

Fig. 5. The signals from the fast-response salinity meter for different Froude numbers.

Internal waves generated by Froude numbers greater than 0.23 were significant and appeared clearly visible near surface, floor and behind the body. As the Froude number increased, wavelengths of internal waves increased as well. The activity of IWs in Froude numbers close to 1 was obvious. When the body moved with the critical Froude number, the wake of the body tended to become turbulent and thus the activity of the internal waves generated by the motion of the body decreased. The turbulent wake could be perturbed by increasing distance from the flow, as shown in Fig. 4 (b). These results were consistent with the findings of Hopfinger et al. Figure 5 shows an example of the signals from the fast-response salinity meter for different Froude numbers.

By approaching critical Froude number, the activity of the internal waves increased and the length and height of these waves rose.

Figure 6 compares the activity of the internal waves for Froude numbers 0.16 and 1.15. As shown in Fig. 6 , by approaching the critical Froude number, the activity of the internal waves increased, and this was also evident in the recorded images, as shown in Figures 3 and 4.

The results obtained from both shadowgraph imaging technique and the signals from a fast response salinity meter were compared. Then the height of the internal waves at the sensor location was evaluated. Figure 7 shows the height of the internal waves for different scenarios resulting from these two techniques.

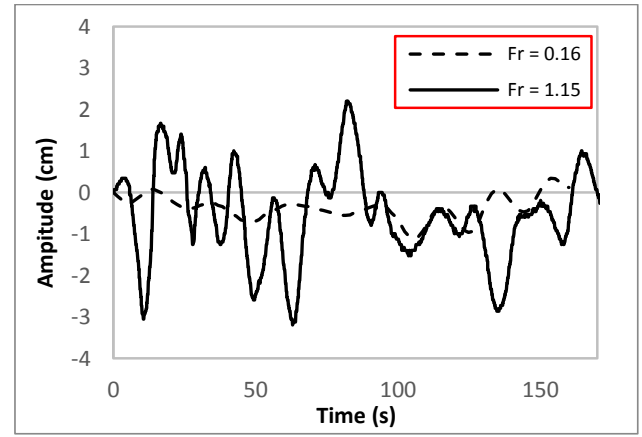

Fig. 6. The activity of the internal waves for $\mathrm{Fr}$ $=0.16$ and $\mathrm{Fr}=1.15$.

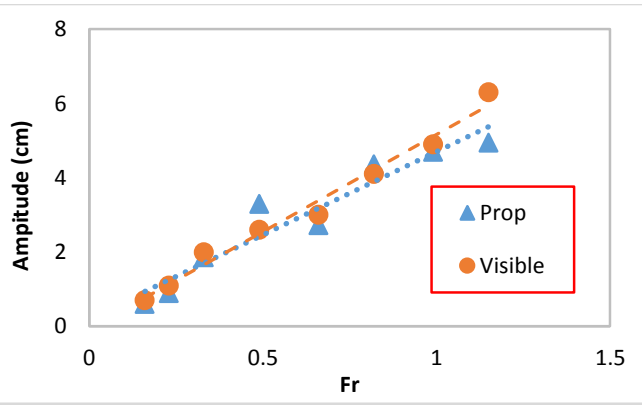

Fig. 7. Comparison of the results of the signals from a fast-response salinity meter and the observations recorded by the shadowgraph imaging technique for the height of internal waves.

As shown in the figure above, there was little error between the recorded signals and the observations of the shadowgraph technique.

\subsection{Validation of Experiments}

In order to validate the experiments performed, the results from this study were compared with those found by Bidokhti et al. (2016) suggesting the same quantized properties. The comparison results together with the linear theory (Eq. 1) are presented in Fig. 8. The obtained results are in good agreement with theory and the study of Bidokhti et al. (2016), as shown in the Fig. 8.

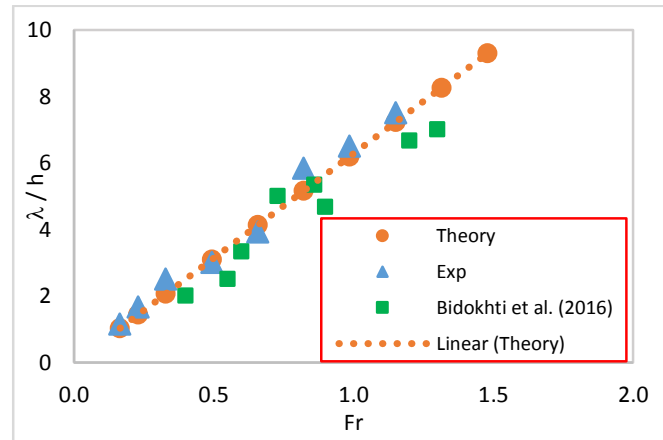

Fig. 8. Horizontal wavelength variations of internal waves quantized by body thickness in terms of Froude number along with linear theory graph and comparison with the experimental results of Bidokhti et al. (2016).

\subsection{The statistical distribution of the recorded signals}

In this section, the statistical distribution of the signals at different Froude numbers are investigated. Based on the received signals, the probability density function (PDF) can be calculated for fitting. For this purpose, a general approach is to approximate the signal to a known PDF and this function must be tested based on each of the parameters of the default model and the best parameter value of the default model is fitted to data. Another approach is the kernel density estimation (KDE) which is a nonparametric way to estimate the PDF of a random variable. If the parametric approaches cannot accurately fit the data or if there is no assumption of 
signal distribution type, then the kernel distribution function will be approximated. This can be defined by a smoothing function and bandwidth (h) that controls the level of smoothing applied to estimate the PDF according to Eq. (12):

$\hat{f}_{h}(x)=\frac{1}{n h} \sum_{i=1}^{n} K\left(\frac{x-x_{i}}{h}\right) ;-\infty<x<\infty$

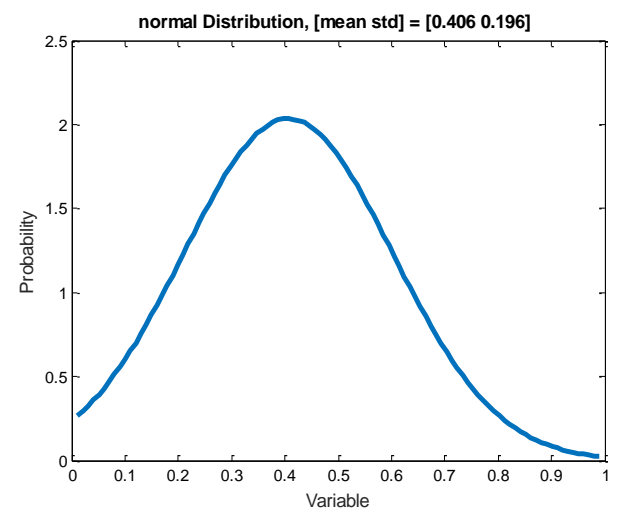

Fig. 9. Matching signal data in the time domain for $\mathrm{Fr}=\mathbf{0 . 3 3}$ with normal distribution fitting.

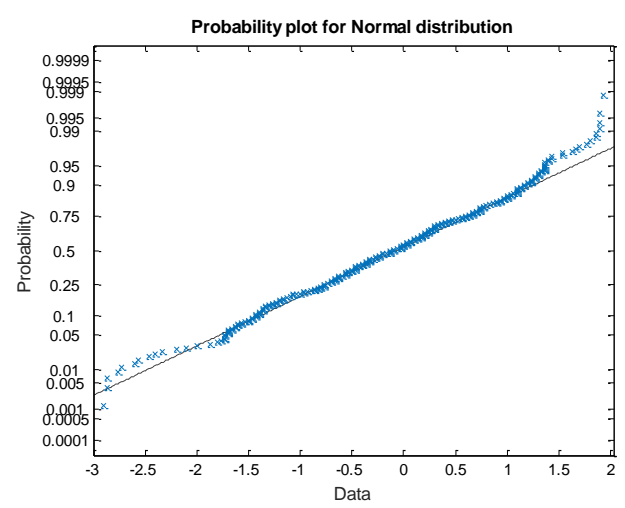

Fig. 10. The probability distribution of received Signals for $\mathrm{Fr}=0.33$.

Where $\mathrm{n}$ is the sample size, $\mathrm{K}($.$) is the kernel$ function, and $\mathrm{h}$ is bandwidth (Li et al. 2011). Figure 9 shows the matching result for signal data in the time domain for $\mathrm{Fr}=0.33$ with normal distribution fitting. If the probability density function is estimated using the KDE, then by analyzing and fitting the sensor signals for different Froude numbers, as shown in Figures 9 and 10 respectively, the statistical distribution function of the signals is very close to the normal probability distribution function. An example of the statistical distribution for $\mathrm{Fr}=0.33$ is shown in Figure 10. No significant similarity was detected in the rest of the probability distribution functions.

\subsection{Frequency Analysis of Recorded Signals}

Spectral analysis of signals is usually performed on each signal class. Using this technique waves and dominant frequencies of the signals are extracted and evaluated. Figure 11 shows some examples of the extracted spectra for different Froude numbers.

Figure 11 shows examples of signals and spectral
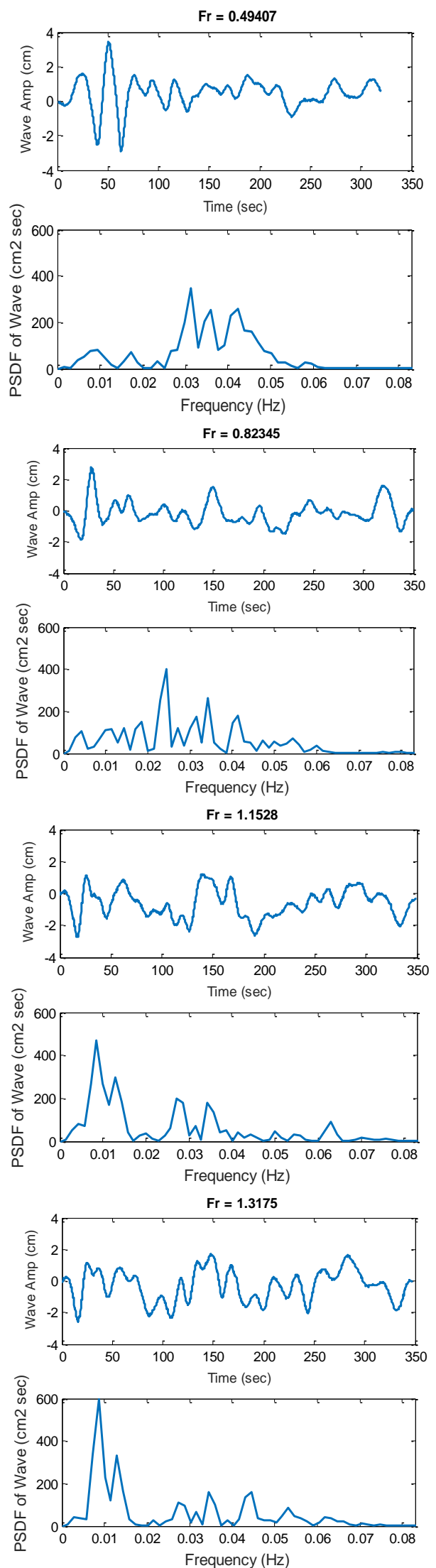

Fig. 11. Frequency spectral density of the recorded signals of the $\mathrm{EC}$ meter for different Froude numbers (the red dashed line indicates the range of buoyancy frequency of the environment). 
analysis for different scenarios. It can be seen that all peak frequencies occurred below the buoyancy frequency of the environment, corresponding to Eq. (4), $f<\omega<N$.

Moreover, as the Froude number increased, the number of peak points in the frequency spectrum increased, indicating the presence of new harmonics of the wave generated. It was also observed that the frequency spectrum grew wider as the Froude number increased. Some frequencies were recorded at different Froude numbers. In the frequency domain, at different Froude numbers, the number of peak points increased as Froude number was close to critical value, indicating an increase in the activity of internal waves, which is consistent with previous studies (Sutherland and Linden 2002; Glushko et al. 1994; Bidokhti et al. 2016; Abdilghanie and Diamessis 2013; Hopfinger et al. 1991; Bidokhti and Britter 2002). Figure 12 compares the frequency spectral densities for different scenarios.

The spectral densities obtained with supercritical Froude numbers were compared with the Kolmogorov turbulent spectra $\left(E(k)=C \varepsilon^{2 / 3} k^{-5 / 3}\right.$

). The slope of the line of the turbulent region was $-\frac{5}{3}$, which is in a good agreement with the frequency analysis of the signal for supercritical Froude numbers (Fig. 13)

By the use of $\mathrm{f}_{\mathrm{m}}$ for different Froude numbers, Eq.s 1 and 5, and substituting $\omega=\frac{f_{m}}{2 \pi}$, as shown in

Fig.12, the wavelength can be calculated as follows:

$\lambda=\frac{\operatorname{Cos} U}{(\theta) f_{m}}$

Where $\mathrm{U}$ is the speed of body, $f_{m}$ is the maximum frequency value and $\theta$ is the wave propagation angle. Figure 14 compares the results of the signal analysis in the empirical Eq. (13) and the linear theory (Eq. 6).

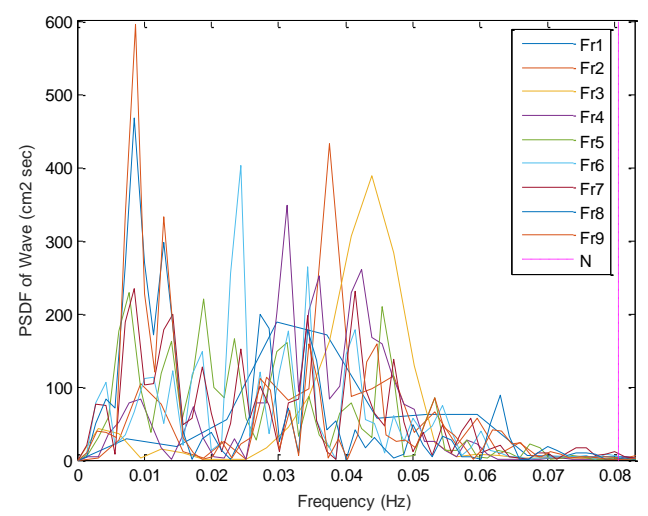

Fig. 12. A comparison of the frequency spectral densities of the signals recorded by ECmeter for different Froude numbers.
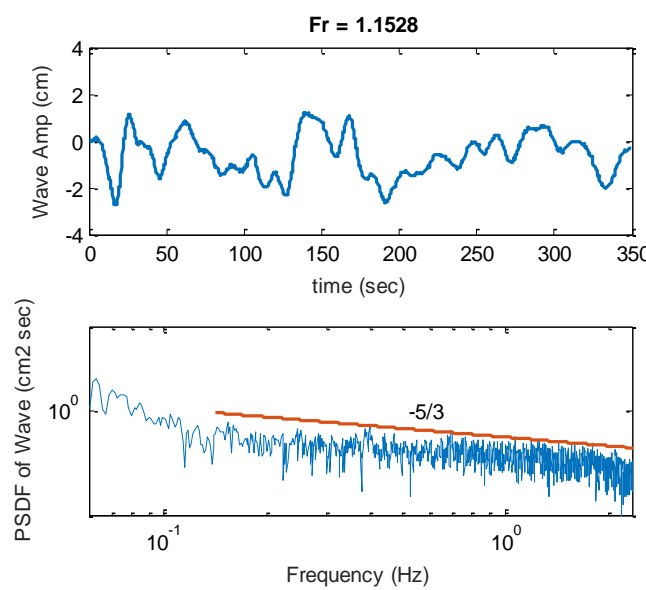

Fig. 13. The logarithmic diagram of the turbulence and slope of the line for $\mathrm{Fr}=1.15$.

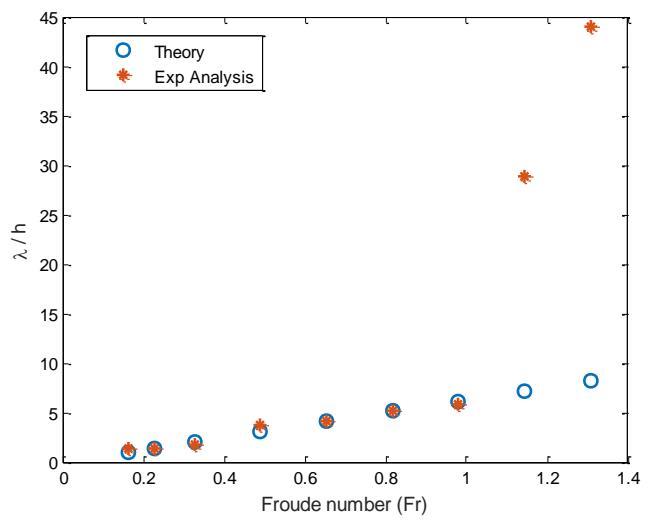

Fig. 14. Comparison of the wavelengths obtained by the empirical relation and the linear theory.

It could be clearly seen in Fig. 14 that the presented relation was used for Froude numbers lower than critical value $(\mathrm{Fr}=1)$, and, further experiments should be performed for Froude numbers higher than one. It should be noted that in nature, most states occur in Froude numbers lower than critical value.

Figure 15 shows how wave energy changes for different Froude numbers. Assessment of the energy values of internal waves obtained from the frequency analysis of the received signals showed that the sum of the maximum values of each signal increased as Froude number increased and after the critical Froude number was reached due to turbulence, the energy of the waves was damped.

\section{CONCLUSION}

In this study, the motion of a cylindrical body in a stratified fluid for different Froude numbers was investigated. In the experiments preformed, the motion of a cylindrical body in a continuously stratified fluid showed that the horizontal wavelength of internal waves behind the body increased with increasing Froude and Reynolds number of the flow as the theory predicted. Of course, this observation continued until Froude numbers reached close to critical value, and then for 
the supercritical flow a turbulent wake appeared behind the body, which collapsed in a short time. Other internal waves would propagate out of collapsing turbulent wake of the body. This study showed that the internal waves generated, remained in the channel for a long period of time.

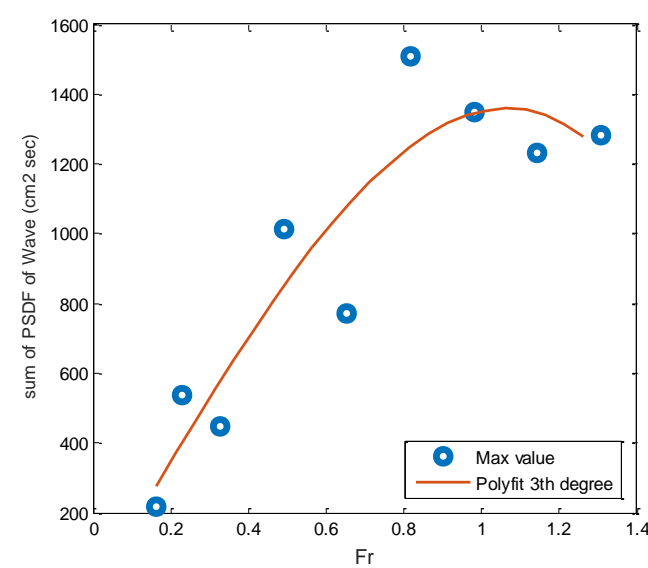

Fig. 15. The sum of the maximum energy values of the internal waves for different Froude numbers.

A fast-response salinity meter was used to analyze the signals behind a cylindrical body which could accurately extract the activity of internal waves, the height of these waves and their wavelengths.

In this study, using $f_{m}$ s extracted for different Froude numbers and applying them to the equations in previous studies, a logical relation was established between the velocity of the body and the wavelength generated, before reaching critical Froude number.

To some extent, the characteristics of the moving body could also be estimated by using the signals recorded by a fast-response salinity meter, the buoyancy frequency of the environment, and the Eq $\frac{U}{N h}=\frac{\lambda}{2 \pi h}$

Therefore, the results of these experiments suggest that such a facility (flow channel with density stratification) with larger dimensions can be used to perform large-scale experiments or to analyze distant internal waves. It is also estimated that the motion of bodies in the fluid with density stratification caused the least mixing and its density profile can be used in numerous experiments for a long period of time.

\section{REFERENCES}

Abdilghanie, A. M. and P. J. Diamessis(2013). The internal gravity wave field emitted by a stably stratified turbulent wake. Journal of Fluid Mechanics 720, 104-139.

Aguilar, D. A., B. R. Sutherland and D. J. Muraki(2006). Laboratory generation of internal waves from sinusoidal topography. Deep Sea Research Part II: Topical Studies in Oceanography 53(1-2), 96-115.
Bidokhti, A. A. (2016). Flow Visualization of Internal Waves and Wakes of a Streamlined Body in a Stratified Fluid. Journal of Applied Fluid Mechanics 9(2).

Bidokhti, A. and R. Britter (2002). A large stratified shear flow water channel facility. Experiments in Fluids 33(2), 281-287.

Bonneton, P., J. M. Chomaz and E. J. Hopfinger, (1993). Internal waves produced by the turbulent wake of a sphere moving horizontally in a stratified fluid. Journal of Fluid Mechanics 254, 23-40.

Dotsenko, S. F. and V. M. Savoskin(1994). Generation of internal waves by moving nonstationary disturbances in a real stratified ocean. Physical Oceanography 5(5), 335-347.

Ekman, S. (1904). Die Phyllopoden, Cladoceren und freischwebenden Copepoden der nordschwedischen Hochgebirage. Ein Beitrag Zur Tiergeographie, Bologie und Systematik der arktischen, nordschwedischen und mitteleuropaischen. Arten Zool Jb Okol. Geog. Tiere 21: 1-170.

Filonov, A. E. and A. Trasviña (2000). Internal waves on the continental shelf of the Gulf of Tehuantepec, Mexico. Estuarine, Coastal and Shelf Science, 50(4), 531-548.

Gill, A. E. (2016). Atmosphere-ocean dynamics. Elsevier.

Glushko, G. S., A. G. Gumilevskii and V. I. Polezhaev (1994). Evolution of the turbulent wakes of spherical bodies in stably stratified media. Fluid Dynamics 29(1), 10-16.

Hopfinger, E. J., J. B. Flor, J. M. Chomaz and P. Bonneton (1991). Internal waves generated by a moving sphere and its wake in a stratified fluid. Experiments in Fluids 11(4), 255-261.

Leder, N. (2002). Wind-induced internal wave dynamics near the Adriatic shelf break. Continental Shelf Research 22(3), 445-463.

Li, X., M. Yu, Y. Liu and X. Xu (2011, September). Feature Extraction of Underwater Signals Based on Bispectrum Estimation. In 2011 7th International Conference on Wireless Communications, Networking and Mobile Computing (pp. 1-4). IEEE

Martin, A. J. (1997). Laboratory simulation and numerical modelling of the kinematics of oceanic internal waves. Ph.D. thesis, The University of Edinburgh, Edinburgh, UK.

Meunier, P., S. Le Dizès, L. Redekopp and G. R. Spedding (2018). Internal waves generated by a stratified wake: experiment and theory. Journal of Fluid Mechanics, 846, 752-788.

Mitkin, V. V. and Y. D. Chashechkin (2000). Experimental Investigation of the Velocity Field near a Cylinder in a Contonuously Stratified Fluid. Fluid Dynamics 35(5), 642651. 
M. Akbarnejadbaie et al. / JAFM, Vol. 13, No. 4, pp. 1253-1262, 2020.

Ohya, Y., T. Uchida and T. Nagai (2013). Near wake of a horizontal circular cylinder in stably stratified flows. Open Journal of Fluid Dynamics 3(4), 311.

Schlosser, T. L., N. L. Jones, R. C. Musgrave, C. E. Bluteau, G. N. Ivey and A. J. Lucas (2019). Observations of Diurnal Coastal-Trapped Waves with a Thermocline-Intensified Velocity Field. Journal of Physical Oceanography 49(7), 1973-1994.

Stevenson, T. N., D. Kanellopulos and M. Constantinides (1986). The phase configuration of trapped internal waves from a body moving in a thermocline. Applied Scientific Research 43(2), 91-105.
Sutherland, B. R. and P. F. Linden (2002). Internal wave excitation by a vertically oscillating elliptical cylinder. Physics of Fluids 14(2), 721731.

Swain, S. K. and K. Trinath (2012). Non-Acoustic Detection of Moving Submerged Bodies in Ocean. International Journal of Innovative Research and Development (ISSN 2278-0211), 1(10), 361-372.

Thorpe, S. A. (2005). The turbulent ocean Cambridge University Press.

Wong, A. B. D., R. W. Griffiths and G. O. Hughes (2001). Shear layers driven by turbulent plumes. Journal of Fluid Mechanics 434, 209241. 